\title{
Experimental investigation of the failure envelope of unidirectional carbon-epoxy composite under high strain rate transverse and off-axis tensile loading
}

\author{
Peter Kuhn a , Marina Ploeckl, and Hannes Koerber \\ Institute for Carbon Composites, Technische Universitaet Muenchen, Faculty of Mechanical Engineering, Boltzmannstrae \\ 15, 85748 Garching bei Muenchen, Germany
}

\begin{abstract}
The mechanical response of the carbon-epoxy material system HexPly IM7-8552 was investigated under transverse tension and combined transverse tension / in-plane shear loading at quasi-static and dynamic strain rates. The dynamic tests of the transverse tension and off-axis tension specimens were carried out on a split-Hopkinson tension bar system, while the quasi-static reference tests were performed on a standard electro-mechanical testing machine. Digital image correlation was used for data reduction at both strain rate regimes. For the high rate tests, the strain rate in loading direction was adjusted to reach approximately the same strain rate value in the fracture plane for each specimen. The measured axial strengths were transformed from the global coordinate system into the combined transverse tension-shear stress space of the material coordinate system and compared with the Puck Mode A criterion for inter-fibre failure. A good correlation between the experimental data and the predicted failure envelopes was found for both investigated strain rate regimes.
\end{abstract}

\section{Introduction}

Over the past years, the number of applications in which fibre reinforced polymer matrix composites (FRPMCs) are used in primary automotive (e.g. BMW i-project) and aeronautical (e.g. Airbus A350 and Boeing 787) structures has significantly increased. In both areas of applications, components may be subjected to high speed loading events such as crash or foreign object impact. Therefore strain rate effects should be captured by advanced composite material models to predict initiation and evolution of damage accurately. The development of corresponding constitutive models and failure criteria requires an improved understanding of the mechanical behaviour of composites under high strain rate loading conditions, especially in the main material directions and under combined stress states. This critically needed data can be provided by high rate loading experiments such as the split-Hopkinson bar test.

In the present work the mechanical properties of a unidirectional (UD) carbon-epoxy composite are investigated under transverse tension and combined transverse tension / in-plane shear loading. Therefore tests at quasi-static and dynamic strain rates using transverse tension and off-axis tension specimens are performed. For the latter specimen type, the authors [1] have observed in a previous study that the strain rate acting in the fracture plane of the off-axis specimens is significantly higher than the applied axial strain rate and additionally depends on the off-axis angles of the specimens. Hence, in this work, the strain rate in the loading direction is adjusted to the reach same strain rate values in the fracture planes, allowing

${ }^{a}$ Corresponding author: kuhn@lcc.mw.tum.de an accurate determination of the corresponding failure envelope.

The dynamic tensile properties of carbon fibre composites with thermoset and thermoplastic matrices in matrix-dominant directions have also been investigated in similar studies by Taniguchi et al. [2,3]. In addition, results from single off-axis and/or transverse tension tests under high strain rates have been published by several authors [4-8]. Dynamic compression tests with off-axis specimens have been performed in different works, as well [9-11]. In the study performed by Koerber et al. [9] the same material as in this work was used for the characterisation of the transverse compression, combined transverse compression and in-plane shear properties under dynamic loading. Together with the data of the present study, a comprehensive data set on the strain rate effect of the matrix dominated compressive and tensile response therefore now exists for this composite material system.

\section{Material and experimental setup}

\subsection{Material and test specimens}

The carbon-epoxy material system HexPly IM7-8552, which is commonly used in primary aerospace components, was selected for this work. In accordance with the specified heat cycle [12], a UD panel with a thickness of $1.5 \mathrm{~mm}$ was manufactured by curing a 12-ply prepreg layup in a hot press. A void content of less than $2 \%$ was determined for this panel by using digital image analysis of photomicrographs.

From the manufactured panel, off-axis tension specimens with fibre orientation angles $\theta=15^{\circ}, 30^{\circ}, 45^{\circ}$ and

This is an Open Access article distributed under the terms of the Creative Commons Attribution License 4.0, which permits unrestricted use, distribution, and reproduction in any medium, provided the original work is properly cited. 
a)

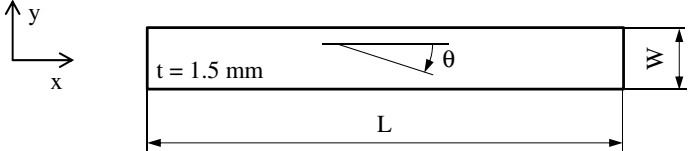

b)

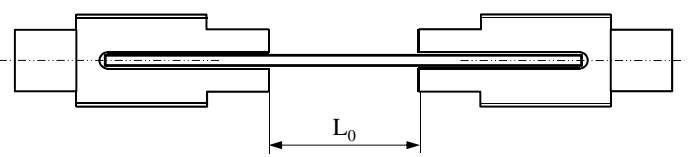

Figure 1. Tension specimen (a) and assembled specimen configuration (b).

transverse tension specimens $\left(\theta=90^{\circ}\right)$ were cut on a water-cooled diamond saw. The nominal dimension of the $15^{\circ}$ off-axis specimens were $72 \times 8 \times 1.5 \mathrm{~mm}^{3}$ and $62 \times 8 \times 1.5 \mathrm{~mm}^{3}$ for all other specimens (Fig. 1a). To attach the specimens to the split-Hopkinson tension bar system, slotted steel adapters with an M12 $\times 1.25$ outside thread were developed. All specimens were glued into the adapters using the structural adhesive 3M ScotchWeld DP 490, while proper alignment of the assembled configuration was ensured by a high accuracy bonding jig. The dimension of the free specimen length $\mathrm{L}_{0}$ between the two adapters was chosen to obtain a section where no fibre is attached to either of the two adapters. Thus for the $15^{\circ}$ off-axis specimens $\mathrm{L}_{0}=30 \mathrm{~mm}$ was used and for all other specimens $\mathrm{L}_{0}=20 \mathrm{~mm}$ was found to be sufficient.

The assembled specimen configuration is shown in Fig. 1b. The specimens were prepared by applying a random black-on-white speckle pattern to enable the use of digital image correlation (DIC) measurement. The fineness of the pattern was adapted to the available image resolutions of the recording camera systems. To ensure that the comparison between the quasi-static and dynamic test results is not affected by size or clamping effects, the same specimen type was used for both test configurations.

\subsection{Quasi-static experimental setup}

The quasi-static reference tests were peformed on a standard electro-mechanical testing machine (Hegewald \& Peschke Inspect Table 100). The adapters of the assembled specimens were screwed into inserts, which in turn were connected to the test machine by a bolt construction (Fig. 2). A constant displacement rate of $0.5 \mathrm{~mm} \cdot \mathrm{min}^{-1}$ was chosen. The GOM ARAMIS-4M system was used for DIC measurement of the three-dimensional in plane strain field. It consisted of two CCD cameras with a resolution of $1728 \times 2352$ pixel $^{2}$, adjusted to capture a measuring volume of $35 \times 26 \mathrm{~mm}^{2}$. The frame rate was 1 frame per second (fps) with a shutter speed of $60 \mathrm{~ms}$.

\subsection{Dynamic experimental setup}

The high strain rate tests were carried out on a splitHopkinson tension bar (SHTB) system. The setup, that is illustrated in Fig. 3, is based on a novel concept proposed by Gerlach et al. [13], using a U-shaped striker-bar.

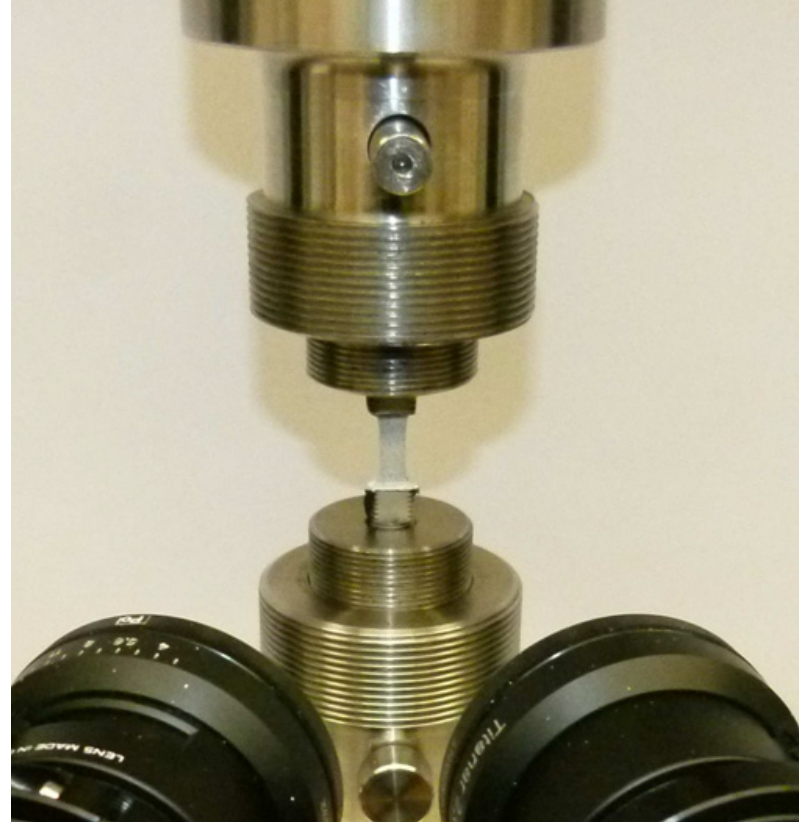

Figure 2. Quasi-static tension test setup.

In this work, a configuration of titanium $\varnothing 20 \mathrm{~mm}$ loading-, Ø16 mm incident- and $\varnothing 16 \mathrm{~mm}$ transmissionbars with lengths of $2.15,3$ and $1.8 \mathrm{~m}$, respectively, was chosen. The striker-bar had a length of $0.5 \mathrm{~m}$. A ring of $2 \mathrm{~mm}$ thick silicon rubber, wrapped around the impact flange at the end of the loading bar, was used for pulse shaping. The resulting ramp-shaped pulse was ideally suited for the predominantly linear stress-strain behaviour [14], which was expected for the dynamic test due to the low plastic strains observed during the quasi-static material characterisation.

For the determination of the two-dimensional strain field using DIC, the deformation of the specimen was monitored by a single Photron SA5 high speed camera. Due to the fact that achievable frame rate and image resolution are contrary setup parameters of the camera, a suitable compromise had to be found for each specimen type. The chosen parameters are listed in Table 1.

\section{Data reduction}

\subsection{Global coordinate system}

Initially, the data reduction was done in the global coordinate system where $x x$ coincides with the loading direction of the assembled specimen configuration. In the case of the quasi-static tests, the nominal axial stress $\sigma_{x x}$ was therefore calculated by dividing the load $F$, measured from the load-cell of the testing machine, by the crosssection $\mathrm{A}_{s}$ of the specimen. For the high rate tests, $\sigma_{x x}$ was determined according to the classic split-Hopkinson pressure bar analysis (SHPBA). According to Gray [15], the specimen stress can be calculated as

$$
\sigma_{x x}=A_{b} E_{b} \varepsilon_{T} / A_{s}
$$

where $\mathrm{A}_{b}, \mathrm{E}_{b}$ and $\varepsilon_{b}$ are the cross-section, Youngs modulus and the measured elastic strain wave of the transmission 


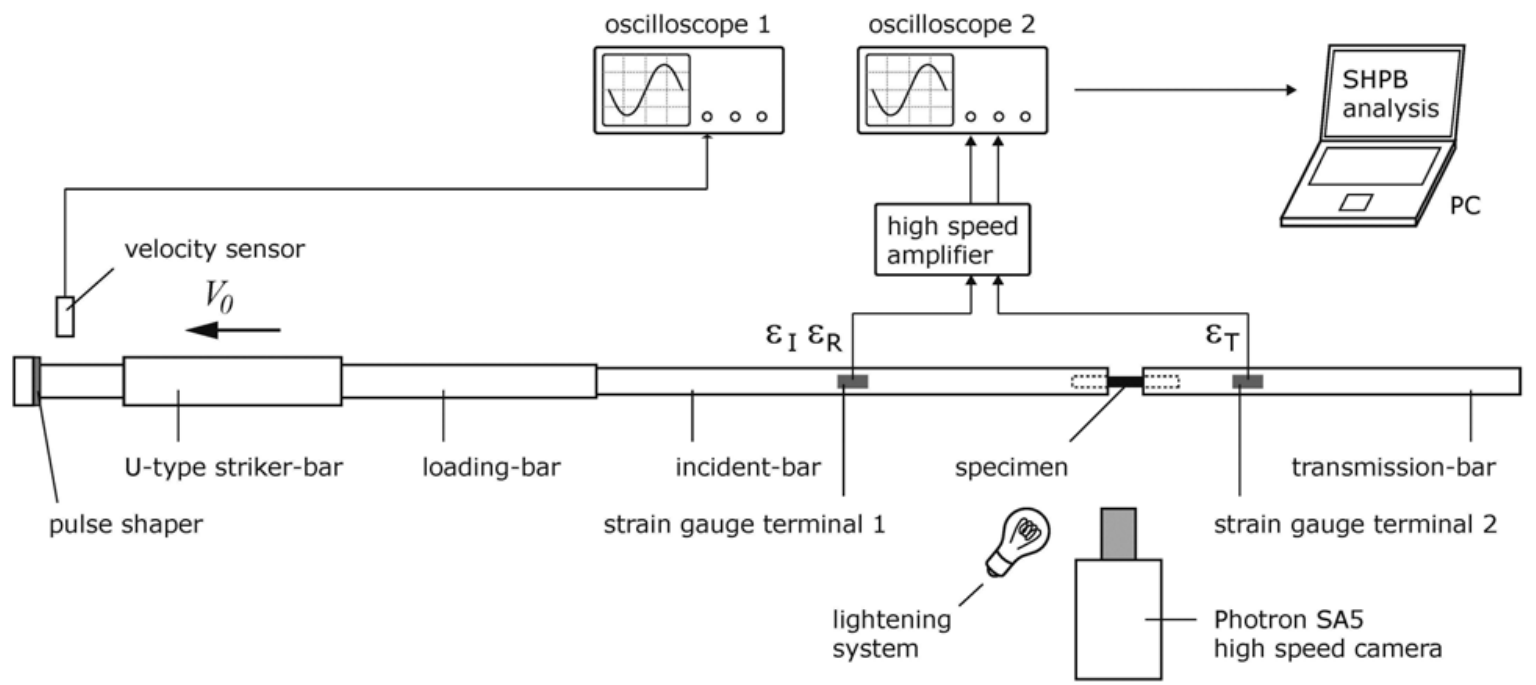

Figure 3. Split-Hopkinson tension bar setup for dynamic tests.

Table 1. Setup parameters of high speed camera.

\begin{tabular}{lll}
\hline Specimen type & $\begin{array}{l}\text { Frames per second } \\
{\left[\mathrm{s}^{-1}\right]}\end{array}$ & $\begin{array}{l}\text { Resolution } \\
{\left[\text { pixel }^{2}\right]}\end{array}$ \\
\hline $15^{\circ}$ & 186.000 & $392 \times 96$ \\
$30^{\circ}$ & 186.000 & $256 \times 112$ \\
$45^{\circ}$ & 300.000 & $192 \times 80$ \\
$90^{\circ}$ & 300.000 & $192 \times 80$ \\
\hline
\end{tabular}

Table 2. ARAMIS analysis parameters.

\begin{tabular}{|c|c|c|}
\hline & Quasi-static & High rate \\
\hline Facet size $\left[\mathrm{pixel}^{2}\right]$ & $17 \times 17$ & $10 \times 10$ \\
\hline Facet step $\left[\right.$ pixel $\left.^{2}\right]$ & $15 \times 15$ & $5 \times 5$ \\
\hline Computation size $\left[\right.$ facets $\left.^{2}\right]$ & $5 \times 5$ & $5 \times 5$ \\
\hline
\end{tabular}

bar. The in-plane strain field $\left\{\varepsilon_{x x}, \varepsilon_{y y}, \gamma_{x y}\right\}^{T}$ of the global coordinate system was obtained for all tests from DIC software GOM ARAMIS. To avoid calculation errors of the DIC at the specimen edges, an area of $5 \times 5 \mathrm{~mm}^{2}$ in the specimen centre was defined for the deformation analysis. The DIC analysis parameters were chosen accordingly to the resolutions of the camera images and are given in Table 2.

The strain component in loading direction was further used for the calculation of axial specimen strain rate $\dot{\epsilon}_{x x}$ by deriving $\varepsilon_{x x}$ with respect to time:

$$
\dot{\epsilon}_{x x}=d \varepsilon_{x x} / d t
$$

\subsection{Material coordinate system}

For the determination of material failure envelopes, it is necessary that the stress-strain response, calculated in the global coordinate system is transformed into the local, material system. For a transverse isotropic material, the orientation along the fibres is denoted as 1-direction and the 2-direction coincides with the in-plane transverse direction. According to Schuermann [16], the stress and strain components of the loading coordinate system can be transformed into the material coordinate system by

$$
\begin{gathered}
\sigma_{22}=\sigma_{x x} \sin ^{2} \beta \\
\tau_{12}=-\sigma_{x x} \sin \beta \cos \beta \\
\gamma_{12}=-\varepsilon_{x x} \sin 2 \beta+\varepsilon_{y y} \sin 2 \beta+\gamma_{x y} \cos 2 \beta
\end{gathered}
$$

where $\beta$ denotes the transformation angle, consisting of the initial off-axis angle $\theta$ and the fibre rotation angle $d \theta$, that occurs during the test due to the extension shear coupling effect in off-axis specimens [17]. The fibre rotation angle can be obtained from DIC software GOM ARAMIS. In case of tensile tests, as performed in this study, the fibres in off-axis specimens tend to orientate in loading direction and therefore the actual transformation angle is

$$
\beta=\theta_{0}-d \theta
$$

The shear strain rate $\dot{\gamma}_{12}$ can be calculated analogous as for the axial strain rate by deriving the corresponding strain value with respect to time:

$$
\dot{\gamma}_{12}=d \gamma_{12} / d t
$$

\section{Results and discussion}

For each specimen type and strain rate regime, three valid tests were performed still allowing a statistical treatment while keeping the experimental efforts within reasonable limits. A test was declared as valid when fracture occurred in the free specimen section.

As observed in a previous study [1], for an accurate comparison of the strain rate effects the strain rates acting in the failure plane should be the same for all tested specimens. To meet this requirement, the axial strain rate had to be increased with increasing off-axis angle of the specimens to capture the corresponding shift from an in-plane shear to a transverse tensile material response. Hence, for the $15^{\circ}, 30^{\circ}$ and $45^{\circ}$ off-axis specimens, the 

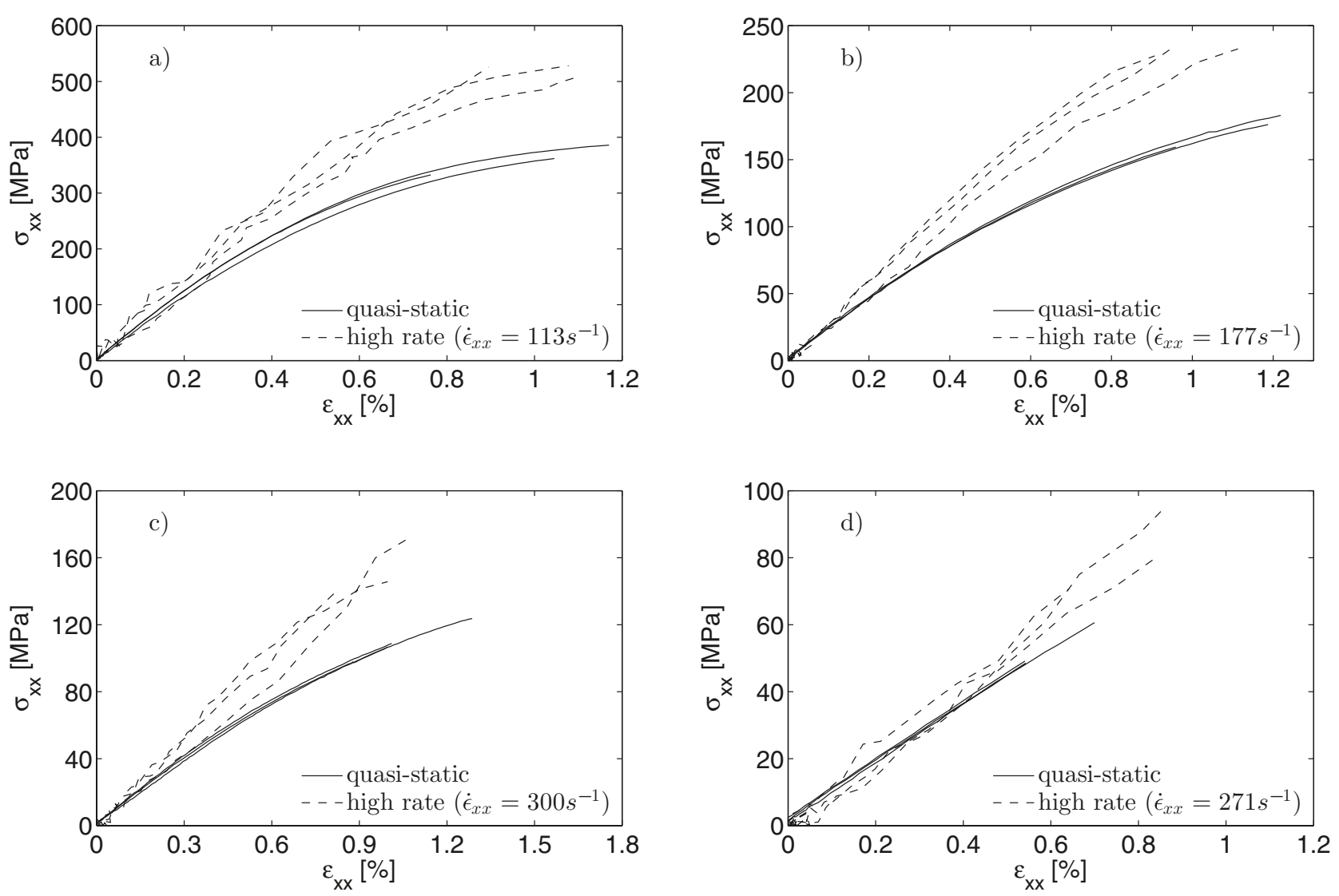

Figure 4. Quasi-static and high rate axial stress-strain curves for: (a) $15^{\circ}$ off-axis tension, (b) $30^{\circ}$ off-axis tension, (c) $45^{\circ}$ off-axis tension and (d) transverse tension.

axial strain rate was adjusted to approximately $110 \mathrm{~s}^{-1}$, $180 \mathrm{~s}^{-1}$ and $300 \mathrm{~s}^{-1}$, respectively, to reach a shear strain rate of $350 \mathrm{~s}^{-1}$. In case of the transverse tension specimens, the axial strain rate is the governed value. Due to the striker-bar velocity limit of the used split-Hopkinson tension bar system, together with the chosen specimen geometry, the attainable strain rate for the $90^{\circ}$ specimens was $271 \mathrm{~s}^{-1}$ and therefore slightly lower than the target value.

The axial stress-strain curves of the off-axis tension and transverse tension specimens for both investigated strain rate regimes are plotted in Fig. 4. All off-axis loaded specimens show non-linear stress-strain behaviour at quasi-static test conditions and a tendency to more linear stress-strain curves under dynamic loading. For the transverse specimens, the stress-strain characteristic is linear at both strain rate regimes. A pronounced strain rate effect on the measured axial strength occurs for all tested specimen configurations. In detail, the strength increases by $45 \%, 34 \%, 35 \%$ and $32 \%$ for the $15^{\circ}, 30^{\circ}$, $45^{\circ}$ and $90^{\circ}$ specimens, respectively, and therefore an approximately constant strain rate effect is found. The measured properties are summarized in Table 3.

The quasi-static and dynamic in-plane shear behaviour of the $15^{\circ}, 30^{\circ}$ and $45^{\circ}$ off-axis specimens, which was calculated from Eqs. (4) and (5), can be seen for representative curves in Fig. 5. The initial part of the shear stress- shear strain curve is similar for both strain rate regimes and seems to be independent of the respective fiber orientation angle. However, the in-plane shear strength value is significantly affected by the superposed transverse stress ratio from Eq. (3).

Figure 6 shows the quasi-static and dynamic failure envelope for combined transverse tension/in-plane shear loading. The pure in-plane shear values were taken from Koerber et al. [9]. They found an increase of $42 \%$ for the in-plane shear strength under similar dynamic loading rates as realized in the present work. For the transverse tension specimens, pronounced scatter in the strength was found for both strain rate regimes. Nevertheless, the calculated mean value for quasi-static loading coincides well with the transverse strength listed in the HexPly IM78552 data sheet [12].

The Puck Mode A failure envelope for twodimensional inter-fibre failure [18], that accounts for the combined transverse tension and in-plane shear stress state in the fracture plane, was constructed for the quasistatic results. For the construction of the dynamic failure envelope an average strength increase of $37 \%$ was used. It can be seen, that the experimental data and the predicted failure envelopes correlate well for both investigated strain rate regimes. For the $15^{\circ}$ off-axis specimens, the predicted strength seems to be slightly over-predictive. A possible explanation for this could be that due to the extension-shear coupling effect, which is most pronounced for this specimen type, premature failure is induced by stress concentrations close to the adapters. It is assumed, however, that with the used specimen configuration, stress 
Table 3. Quasi-static and high rate off-axis tension and transverse tension properties.

\begin{tabular}{|c|c|c|c|c|c|c|}
\hline Loading rate & $\begin{array}{l}\text { Fibre angle } \\
\theta\left[{ }^{\circ}\right] \\
\end{array}$ & & $\begin{array}{l}\text { Ultimate strength } \\
{[\mathrm{MPa}]}\end{array}$ & $\begin{array}{l}\text { Shear angle } \\
\mathrm{d} \theta\left[^{\circ}\right]\end{array}$ & $\begin{array}{l}\text { Axial strain rate } \\
\dot{\varepsilon}_{x x}\left[s^{-1}\right] \\
\end{array}$ & $\begin{array}{l}\text { Shear strain rate } \\
\dot{\gamma}_{12}\left[s^{-1}\right]\end{array}$ \\
\hline \multirow[t]{12}{*}{ quasi-static } & \multirow[t]{3}{*}{$15^{\circ}$} & mean & 360 & 1.56 & $2.1 \cdot 10^{-4}$ & $7.8 \cdot 10^{-4}$ \\
\hline & & STDV & 22 & 0.32 & $2.8 \cdot 10^{-5}$ & $1.8 \cdot 10^{-4}$ \\
\hline & & $\mathrm{CV}[\%]$ & 6.1 & 20.5 & 13.3 & 15.3 \\
\hline & \multirow[t]{3}{*}{$30^{\circ}$} & mean & 173 & 0.82 & $2.9 \cdot 10^{-4}$ & $6.0 \cdot 10^{-4}$ \\
\hline & & STDV & 10 & 0.09 & $7.7 \cdot 10^{-6}$ & $4.2 \cdot 10^{-5}$ \\
\hline & & $\mathrm{CV}[\%]$ & 5.8 & 11.0 & 2.7 & 7.0 \\
\hline & \multirow[t]{3}{*}{$45^{\circ}$} & mean & 113 & 0.35 & $2.6 \cdot 10^{-4}$ & $3.8 \cdot 10^{-4}$ \\
\hline & & STDV & 8 & 0.03 & $2.9 \cdot 10^{-5}$ & $3.3 \cdot 10^{-5}$ \\
\hline & & $\mathrm{CV}[\%]$ & 7.1 & 8.6 & 11.3 & 8.6 \\
\hline & \multirow[t]{3}{*}{$90^{\circ}$} & mean & 62 & - & $2.8 \cdot 10^{-4}$ & - \\
\hline & & STDV & 11 & - & $1.7 \cdot 10^{-5}$ & - \\
\hline & & $\mathrm{CV}[\%]$ & 17.7 & - & 5.9 & - \\
\hline \multirow[t]{12}{*}{ high rate } & \multirow[t]{3}{*}{$15^{\circ}$} & mean & 521 & 1.45 & 113 & 358 \\
\hline & & STDV & 9 & 0.20 & 19 & 51 \\
\hline & & $\mathrm{CV}[\%]$ & 1.7 & 13.8 & 16.8 & 14.2 \\
\hline & \multirow[t]{3}{*}{$30^{\circ}$} & mean & 231 & 0.71 & 177 & 337 \\
\hline & & STDV & 3 & 0.05 & 10 & 10 \\
\hline & & $\mathrm{CV}[\%]$ & 1.3 & 7.0 & 5.6 & 3.0 \\
\hline & \multirow[t]{3}{*}{$45^{\circ}$} & mean & 153 & 0.34 & 300 & 410 \\
\hline & & STDV & 14 & 0.04 & 44 & 64 \\
\hline & & $\mathrm{CV}[\%]$ & 9.2 & 11.8 & 14.7 & 15.6 \\
\hline & \multirow[t]{3}{*}{$90^{\circ}$} & mean & 82 & - & 271 & - \\
\hline & & STDV & 10 & - & 13 & - \\
\hline & & $\mathrm{CV}[\%]$ & 12.2 & - & 4.8 & - \\
\hline
\end{tabular}

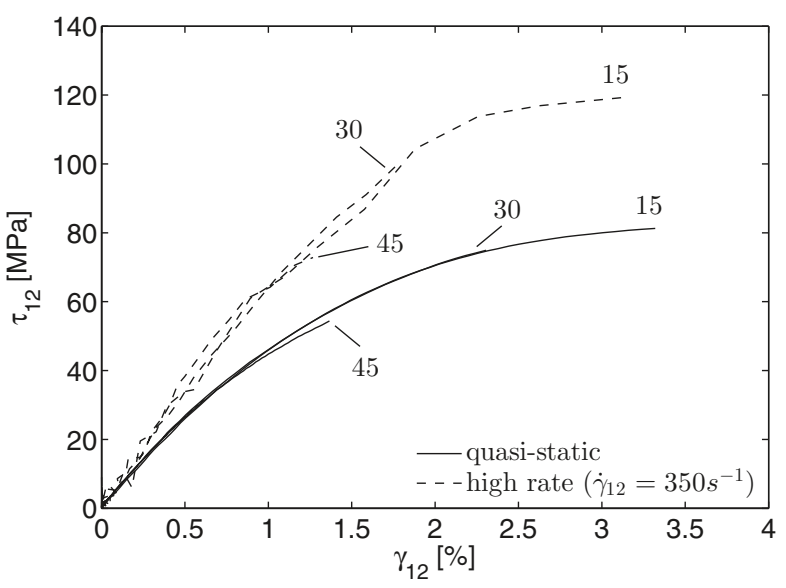

Figure 5. In-plane shear behaviour from off-axis tension tests.

concentrations could be reduced due to the compliance of the adhesive layer, compared to rigid clamping boundary constraints at quasi-static standard tests.

\section{Summary and conclusion}

The mechanical response of the carbon-epoxy material system HexPly IM7-8552 was investigated under transverse tension and combined transverse tension / in-plane shear loading. Tests at quasi-static and dynamic strain rates using transverse tension and off-axis tension specimens

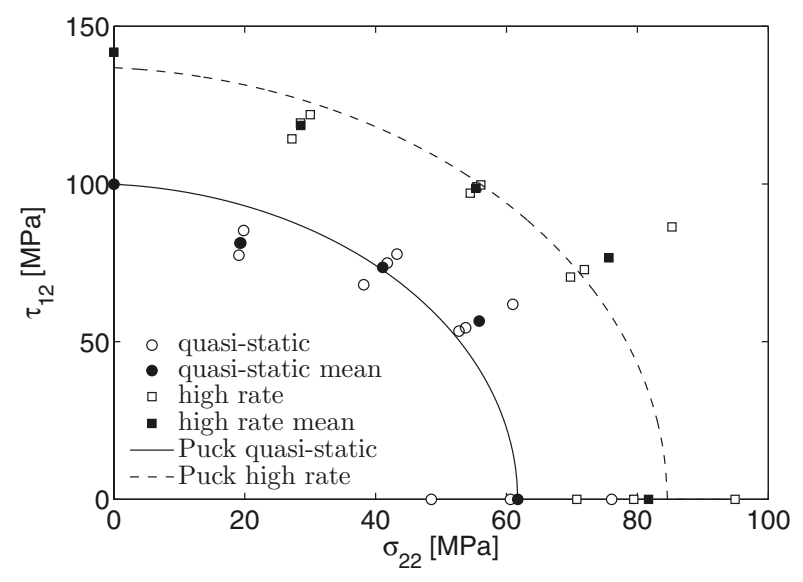

Figure 6. Experimental and predicted inter-fibre failure (IFF) envelope for quasi-static and high rate loading.

were performed. A linear stress strain-strain response was found for the transverse specimens at both strain rates, whereas the off-axis loaded specimens showed nonlinear stress-strain behaviour at quasi-static test conditions and a tendency to more linear stress-strain curves under dynamic loading. The measured axial strengths increased by $45 \%, 34 \%, 35 \%$ and $32 \%$ for the $15^{\circ}, 30^{\circ}, 45^{\circ}$ and $90^{\circ}$ specimens, respectively. An approximately constant strain rate effect was therefore found which can be attributed to same strain rate values in the fracture planes of all 
specimens. This was reached by adjusting the strain rate in the loading direction, accordingly.

In the combined transverse tension/in-plane shear stress space, good correlation between the experimental data and the Puck Mode A IFF failure envelope was observed for both strain rate regimes.

For all specimen configurations, an average increase of $37 \%$ for the ultimate strength under dynamic loading was found. This coincides well with the results for combined transverse compression/in-plane shear loading published by Koerber et al. [9]. They found an average increase of $40 \%$ under similar dynamic loading rates as realized in the present work. A comprehensive data set on the strain rate effect of the matrix dominated compressive and tensile response of HexPly IM7-8552 therefore now exists.

\section{References}

[1] H. Koerber, M. Vogler, P. Kuhn, P.P. Camanho, Proceedings of ECCM16 - 16th European Conference on Composite Materials, Seville, Spain (2014)

[2] N. Taniguchi, T. Nishiwaki, H. Kawada, Advanced Composite Materials 16, 167-180 (2007)

[3] N. Taniguchi, T. Nishiwaki, N. Hirayama, H. Nishida, H. Kawada, Composites Science and Technology 69, 207-213 (2009)

[4] A. Gilat, R.K. Goldberg, G.D. Roberts, Composites Science and Technology 62, 1469-1476 (2002)

[5] S.V. Thiruppukuzhi, C.T. Sun, Composites Science and Technology 61, 1-12 (2001)
[6] J. Harding, L.M. Welsh, Journal of Materials Science 18, 181-1826 (1983)

[7] T. Gómez-del Río, E. Barbero, R. Zaera, C. Navarro, Composites Science and Technology 65, 61-71 (2005)

[8] L.G. Melin, L.E. Asp, Composites: Part A 30, 305-316 (1999)

[9] H. Koerber, J. Xavier, P.P. Camanho, Mechanics of Materials 42, 1004-1019 (2010)

[10] I.M. Daniel, B.T. Werner, J.S. Fenner, Composites Science and Technology 71, 357-364 (2011)

[11] J.L. Tsai, C.T. Sun, Composites Science and Technology 65, 1941-1947 (2005)

[12] Material Data Sheet: HexPly 8852 Product Data (Feb. 2013)

[13] R. Gerlach, C. Kettenbeil, N. Petrinic, International Journal of Impact Engineering 50, 63-67 (2012)

[14] H. Koerber, P.P. Camanho, Composites Part A 42, 462-470 (2011)

[15] G.T. Gray III, ASM Handbook Vol. 8 Mechanical Testing and Evaluation (ASM International, Ohio, 2000) 462-476

[16] H. Schuermann Konstruieren mit Faser-KunststoffVerbunden, 2nd edn. (Springer Verlag, Berlin Heidelberg, 2007)

[17] C.T. Sun, S.P. Berreth, Journal of Composite Materials 22, 766-779 (1988)

[18] A. Puck, H. Schuermann, Composites Science and Technology 58, 1045-1067 (1998) 